\title{
Clinical Audit - What is it and how to do it?
}

\author{
Sandhya K Lokuarachchi \\ Consultant Paediatrician, Karapitiya Teaching Hospital, Galle.
}

There is much concern about the quality of care provided to our patients and the standard of the care givers. Many instances of national publicity and criticism regarding patient care in our hospitals were seen commonly in recent past. Society has become very vigilant and critical about the standard of patient outcomes.

There is no doubt that all attempts should be made to give best quality care to our patients. To achieve this there should be a systematic way of identifying the deficiencies honestly with genuine attempts at improving the shortcomings.

Clinical audit is the process that supports continuous quality improvement programmes [1]. It is one way in which we can work to retain trust and respect of patients, in an increasingly critical and challenging environment. Clinical Audit can demonstrate that real efforts are being made by staff to deliver care of high quality to our patients [2]. Further it also provides a systematic mechanism that compares the care provided to evidence based best practice and assists in identifying the deficiencies in the system and ways of improving them [1].

\section{What is clinical audit?}

Clinical audit is by definition a quality improvement process that seeks to improve patient care and outcomes through systematic review of care against explicit criteria and the implementation of changes. Aspects of the structure, processes and outcomes of care are systematically evaluated against explicit criteria. Changes are implemented where indicated at an individual, team or service level and further monitoring is used to confirm improvement in health care delivery $[1,3]$.

Clinical audit ensures that what should be done is being done. If it is not being done it provides a framework to enable changes to be made to improve the process.

\section{History}

Monitoring of patient outcomes had been carried out as early as 1750 BC, during king Hammurabi of Babylon, with punishments for clinicians for poor performance [3]. However, the first clinical audit is considered to have been carried out by Florence Nightingale during the Crimean war of 1853-5, when improvement of standards following an “ audit” on cleanliness remarkably reduced the mortality rates in hospital patients [4]. Ernest Codman is known as the first true medical auditor following his work on monitoring surgical outcomes in 1912. Since then process of evaluating patient care remained static for many years. First move to integrate clinical audit in professional health care in United Kingdom was the introduction of the white paper, in 1997 [4]. The process which was initially established as a medical audit subsequently evolved into a clinical audit.

\section{Types of clinical audit}

1. Standard based audits: A cycle involving defining standards, collecting data to measure current practice against these standards and implementing necessary changes.

2. Adverse occurrence screening and critical incident monitoring: Often used to review cases with special concern or unexpected outcomes. This reflects the way the team has functioned and helps to learn for future.

3. Peer-review: Individual cases are discussed by peers to determine whether best care was given. Somewhat similar to type 2 above, but might include interesting or unusual cases rather than critical incidents. Recommendations are very often not followed as there is no systematic method to follow.

4. Patient surveys and focus groups: Carried out by obtaining views of patients, regarding the care received. 


\section{Clinical audit process}

The process can be described as a cycle or spiral, where there are stages of a systematic process aimed at improving quality of patient care. As the process continues each cycle aspires to a higher level [4,5].

Audit staff should be identified at the very beginning of the process. Objectives of the audit should be clearly laid down. What is to be learnt from the audit, the problems identified and the desired improvements should be discussed by the audit staff.

\section{Steps in audit process}

Stage 1 - Identify topic / problem to be audited

Topic chosen should be of obvious importance to the team, department and patients. Selection of topics may be based on, presence of identified problems (eg: from complaints or adverse incidents), high volume, high risk or high cost areas of practice, areas with published evidence about clinically effective treatment and availability of clinical guidelines [4,5].

\section{Stage 2 - Define criteria and standards}

Criteria are explicit statements that define what is being measured. It is a measurable outcome of care. Standard is the threshold of the expected compliance for each criterion. This is usually given as a percentage. Recommendations from clinical practice guidelines can be used to develop criteria and standards.

\section{Stage 3 - Method and data collection}

Method or type of audit process is chosen depending on audit objectives. Data collection should be precise and only the essential data should be collected. Data collection may be retrospective, concurrent or prospective $[4,5,6]$. Data may be collected using computerized information or done manually. Consideration should be given to what data will be collected, where data will be available and who will collect the data.
Ethical issues should be given due consideration. Data collected must relate only to the objectives. Patient and staff confidentiality need to be respected.

Stage 4 - Comparison of performance with criteria and standards

Data collected are analysed. Results are compared with criteria and standards. How well the standards were met is concluded. Reasons for any deviation from standards and areas with potential for improvement should be recognized $[4,5,7]$.

Stage 5 - Implementing change

Once the results of audit are analysed and discussed and areas for potential improvement are identified, recommendations for improvement are formulated [4,5]. Action plan should be made for implementing changes. Persons responsible and time frame and the action to be taken should be clearly defined.

At this stage a report should be sent to the clinical audit department where there are well organized clinical audit departments [4,5]. In the absence of a well organized system report should be forwarded to administrators who are responsible for overall patient care at local or national level.

\section{Re-audit and sustaining improvement}

After an agreed period of implementing changes the audit should be repeated [3,4,5]. Same strategies used for the original audit should be used to ensure comparability. The re-audit should reveal that the changes have been implemented and improvement has been made. Depending on achievements further re-audits may be planned. If acceptable quality of care is sustained reauditing can be replaced by some form of monitoring and audit processes reintroduced when indicated.

Results of good clinical audits should be disseminated locally, nationally and internationally when relevant $[3,4,5]$. 


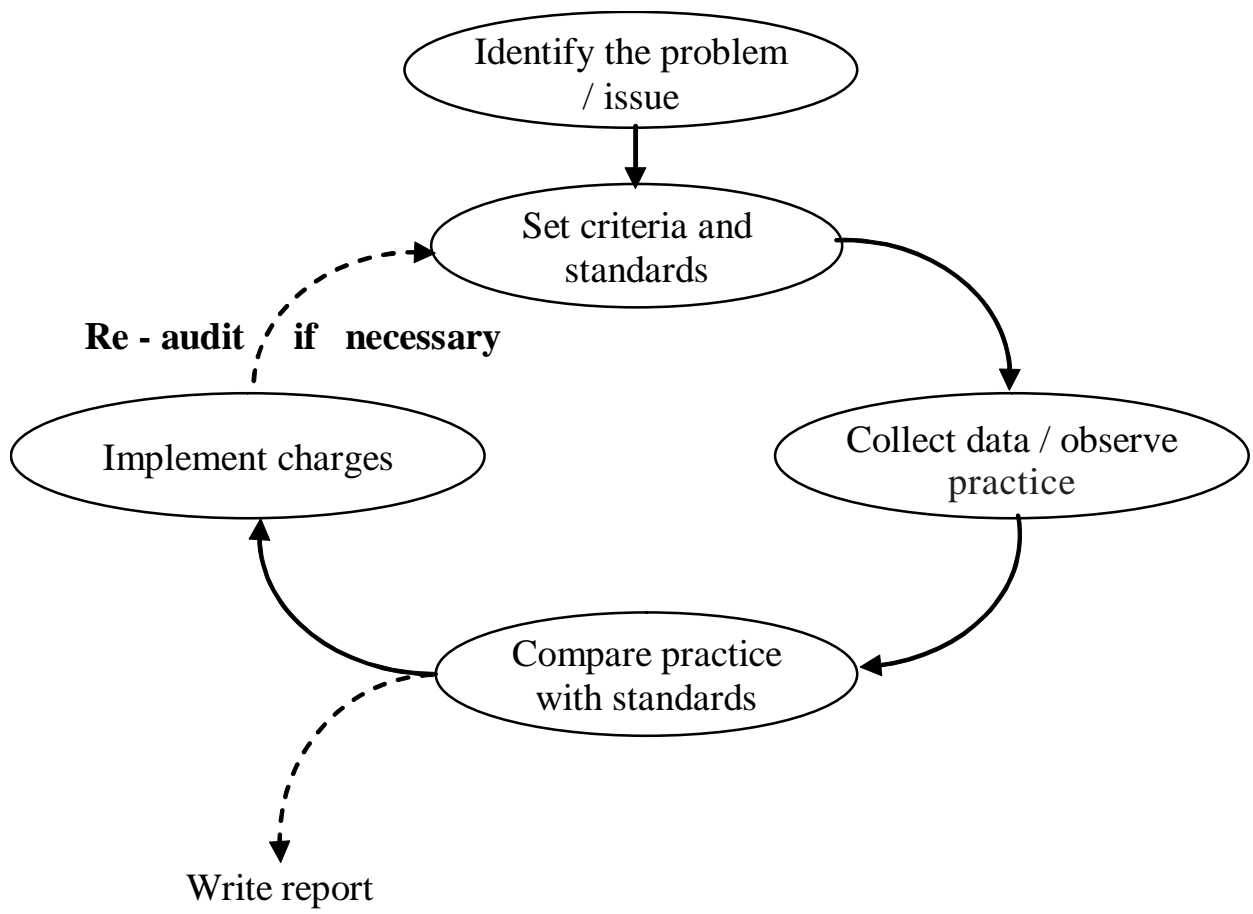

Audit Process

\section{Management of clinical audit process}

In developed countries, within strategic health authorities, clinical audits take place under a supportive organisation - clinical governance [1,3,4]. Clinical governance lead coordinates governance activities within an organization providing health care. Clinical audit lead is responsible for clinical audit strategies, arraigning and implementing audit programmes at local level. Clinical audit lead coordinates formation of Clinical audit committee, which is responsible for overseeing audit programmes and dissemination of results of audits performed. Clinical audit manager is responsible for management and allocation of staff - facilitators and clerks, to deliver the programme within the desired time frame. He is responsible for effective completion of all steps of audit programme. Clinicians involved in delivery of care play a main role in monitoring data collection.

Routine clinical audit programmes should be essential components of good professional practice $[1,4]$. All individuals and professional bodies involved in patient care and the government should be committed to introduce a systematic mechanism to integrate regular clinical audit programmes in health care service of our country.

\section{References}

1. Principles of best practice in clinical audit, National Institute of Clinical Excellence; London: NICE - 2002. [cited 2006 June 23]. Available from http:// www.nelh.nhs.uk/ nice_bpca.asp.

2. Clinical governance and re-validation: the role of clinical audit, Education in Pathology. 2002; 117: 47-50. [cited 2006 July 3]. Available from http://www.rcpath.org / resources/pdf/clinical.pdf

3. Clinical Governance Support Team, A Practical Handbook for Clinical Audit. [cited 2006 June 7]. Available from http://www.cgsupport.nhs.uk/ $\underline{\text { Resources/Clinical_Audit/1@ }}$ $\underline{\text { IntroductionandContents.asp }}$

4. Clinical audit, Wikipedia, the free encyclopedia. [cited 2005 Dec25]. Available from http://.en.wikipedia.org/ wiki/Clinical audit

5. How to do clinical audit - a brief guide, UBHT Clinical Audit Central Office. 2005 [cited2006 July 3]. Available from http://www.ubht.nhs.uk/clinicalaudit/ docs/HowTo/intro.pdf

6. How to collect audit data, UBHT Clinical Audit Central Office.2005. [cited 2006 July 3]. Available from http:/ /www.ubht.nhs.uk/clinicalaudit/docs/HowTo/ DataCollection.pdf

7. How to analyse audit data, UBHT Clinical Audit Central Office 2005. [cited 2006 July 3]. Available from http:/ /www.Ubht.nhs.uk/clinicalaudit/docs/HowTo/ Analysis.pdf 